INPLASY

PROTOCOL

To cite: Guo et al. Acupuncture Combined with PrickingCupping Therapy for Postherpetic Neuralgia: A Protocol of Systematic Review and Meta-analysis. Inplasy protocol 202080092. doi: 10.37766/inplasy2020.8.0092

Received: 22 August 2020

Published: 22 August 2020

Corresponding author: Jun Xiong

xiongjun196071@163.com

Author Affiliation:

Jiangxi University of

Traditional Chinese Medicine

Support: 1050Project:

5141900101

Review Stage at time of this submission: The review has not yet started.

Conflicts of interest:

The authors declare no conflicts of interest.

\section{Acupuncture Combined with Pricking-Cupping Therapy for Postherpetic Neuralgia: A Protocol of Systematic Review and Meta-analysis}

Guo, $\mathrm{H}^{1}$; Xiong, J2; Zhou, XC³.

Review question / Objective: To summarize and evaluate the effects of acupuncture combined with pricking-cupping therapy for postherpetic neuralgia.

Condition being studied: Postherpetic neuralgia(PHN) is a typical neuropathic pain, which is the most common chronic complication of herpes zoster. PHN make patients suffer from paroxysmal or persistent burning pain of varying degrees, cause anxiety, depression, and insomnia. Acupuncture as a traditional treatment in China, has been widely used in the clinical treatment of postherpetic neuralgia. A lot of studies have shown that acupuncture is effective. Cupping is a physical treatment used by acupuncturists or other therapists, which utilize a glass or bamboo cup to create suction on the skin over a painful area or acupuncture point. It is mostly used in Asian and Middle Eastern countries and has been claimed to reduce pain as well as a host of other symptoms. There are two types of cupping. Dry cupping pulls the skin into the cup without drawing blood. In wet cupping the skin is lacerated so that blood is drawn into the cup. Several studies have confirmed the effectiveness of pricking-cupping in the treatment of postherpetic neuralgia.

INPLASY registration number: This protocol was registered with the International Platform of Registered Systematic Review and Meta-Analysis Protocols (INPLASY) on 22 August 2020 and was last updated on 22 August 2020 (registration number INPLASY202080092).

\section{INTRODUCTION}

Review question / Objective: To summarize and evaluate the effects of acupuncture combined with pricking-cupping therapy for postherpetic neuralgia.
Condition being studied: Postherpetic neuralgia(PHN) is a typical neuropathic pain, which is the most common chronic complication of herpes zoster. PHN make patients suffer from paroxysmal or persistent burning pain of varying degrees, cause anxiety, depression, and insomnia. 
Acupuncture as a traditional treatment in China, has been widely used in the clinical treatment of postherpetic neuralgia. A lot of studies have shown that acupuncture is effective. Cupping is a physical treatment used by acupuncturists or other therapists, which utilize a glass or bamboo cup to create suction on the skin over a painful area or acupuncture point. It is mostly used in Asian and Middle Eastern countries and has been claimed to reduce pain as well as a host of other symptoms. There are two types of cupping. Dry cupping pulls the skin into the cup without drawing blood. In wet cupping the skin is lacerated so that blood is drawn into the cup. Several studies have confirmed the effectiveness of pricking-cupping in the treatment of postherpetic neuralgia.

\section{METHODS}

Participant or population: Patients with postherpetic neuralgia.

Intervention: Acupuncture combined with pricking-cupping.

Comparator: The control group was treated with acupuncture, drug, acupuncture plus drugs, acupuncture combined with other traditional Chinese medicine therapies, etc.

Study designs to be included: Study type: Randomized controlled trial.

Eligibility criteria: Subjects: patients with postherpetic neuralgia, age and sex were not restricted. Intervention measures: acupuncture combined with prickingcupping was used as the intervention measures in the treatment group, while acupuncture, drug, acupuncture plus drugs, acupuncture combined with other traditional Chinese medicine therapies was used as the intervention measures in the control group Outcome measures: cure rate, effective rate, inefficiency, pain intensity.

Information sources: Pubmed, Embase, Cochrane Library, Chinese Biomedical Literatures Database(CBM), China National Knowledge Infrastructure (CNKI),
WangFang Database (WF), Chinese Scientifific Journal Database (VIP).

Main outcome(s): Effective rate; pain intensity (VAS, NRS, MPQ, etc.).

Quality assessment / Risk of bias analysis: The methodological quality of each included RCT will be evaluated using the CONSORT 2010 checklist. This process will be carried out by two reviewers independently. Any disagreement will be resolved by discussion or by consulting a third reviewers until consensus is reached.

Strategy of data synthesis: RevMan $\mathbf{5 . 4}$ software (Cochrane Collaboration) was used for the meta-analysis. Dichotomous data were reported as risk ratio (RR) with $95 \%$ confidence intervals $(\mathrm{CI})$, while continuous data were reported as standardized mean difference (SMD) with $95 \%$ Cls. The Higgins $I^{2}$ test was used to test heterogeneity with a significance level set at $50 \%$. If heterogeneity was not significant $\left(I^{2} \leq 50 \%\right)$, the fixed effects model was used for meta-analysis. Otherwise, the random effects model was used $\left(I^{2} \geq 50 \%\right)$. If possible, we investigated the potential explanations for heterogeneity and conducted subgroup analysis.

Subgroup analysis: Subgroup analysis will be conducted for heterogeneity arising from control type (acupuncture, drug, acupuncture plus drugs, acupuncture combined with other traditional Chinese medicine therapies, etc), treatment duration and acupoints.

Sensibility analysis: Sensibility analysis will be conducted to assess the influence of each individual study, leave-one-out sensitivity analysis was performed iteratively by removing one study at a time to confirm that the findings were not influenced by any single study.

Country(ies) involved: China.

Keywords: Postherpetic neuralgia; Acupuncture; Pricking-cupping therapy; 
Randomized controlled trials; Systematic review; Meta-analysis.

Contributions of each author:

Author 1 - Hun Guo - The author drafted the manuscript and did statistical work.

Author 2 - Jun Xiong - Gave the conception and reviewed the manuscript, provided feedback for the manuscript and do the supervision work.

Author 3 - XingChen Zhou - Contributed to the development of the selection criteria, and the risk of bias assessment strategy. 